\title{
Statistical Fatigue Property of Multi-Layer Amorphous Metal Composites in Axial Loading*
}

\author{
Yanbin ZHANG**, Tatsuo SAKAI** and Kiyoteru MORI*** \\ ${ }^{* *}$ Faculty of Science and Engineering, Ritsumeikan University, \\ 1-1-1 Nojihigashi, Kusatsu, Shiga, 525-8577 Japan \\ E-mail:sakai@se.ritsumei.ac.jp \\ *** Handa Plant, Fuji Heavy Industries Ltd., \\ 1-27, Ushioicho, Handa, Aichi, 475-0032 Japan
}

\begin{abstract}
Statistical fatigue property and fracture mechanism of a Fe-based film amorphous metal, $\mathrm{Fe}_{78} \mathrm{~B}_{13} \mathrm{Si}_{9}$, have been examined in the axial loading. Testing specimens including single-layer and multi-layer types were prepared from a long ribbon of $\mathrm{Fe}_{78} \mathrm{~B}_{13} \mathrm{Si}_{9}$ amorphous metal with dimensions of $25 \mu \mathrm{m}$ in thickness and $50 \mathrm{~mm}$ in width. The high cycle fatigue tests were performed by using electro-hydraulic servo testing machine in tension-tension loading at a frequency of $50 \mathrm{~Hz}$ with the stress ratio of 0.1 . The testing data showed that fatigue lives of the amorphous metal had a large scatter, especially at low stress levels. By applying common Weibull distribution and mixed-mode Weibull distribution, the statistic fatigue property of the specimens was quantitatively analyzed. The fatigue strength $\sigma$ at $N=10^{7}$ was in the range of 135-187 MPa and the fatigue ratio to the tensile strength was found to be $\sigma / \sigma_{\mathrm{B}}=6-9 \%$. The fracture surface morphology of failed specimens observed by SEM showed evidently three main regions as follows: 1) flat area corresponding to the crack initiation and the early growth stages up to penetrate the thickness of the film specimen. 2) rough area with a lot of chevron patterns corresponding to the stable propagation as the penetrated fatigue crack. 3) relatively plain area with vein patterns peculiar to the static fracture surface of amorphous metals.
\end{abstract}

Key words: Fe-Based Amorphous, Amorphous Film, Multi-Layer Specimens, Statistical Fatigue Property, Fractography

\section{Introduction}

Usual metallic materials which have a highly ordered arrangement of atomic structures are crystalline in nature. The first synthesis of an amorphous solid with disordered arrangement of atomic structures was reported in 1960 [1], which was formed by the rapid quenching of an Au-Si alloy with cooling rate up to $10^{6} \mathrm{~K} / \mathrm{s}$. Due to the disordered atomic structures, amorphous metals have many excellent properties such as high tensile strength, superior electromagnetic property, high corrosion resistance and so on [2-4]. In addition, their superiority in electromagnetic and high tensile strength properties renders them the best candidate as core materials of transformers and electric motors [5]. However, such disordered structure materials which are produced directly from the liquid state have to be cooled rapidly to avoid the crystallization. In order to realize the high cooling rate, amorphous metals were produced in the shapes of wires, ultra thin films, and foils in the early studies. Thus, for example, a number of thin films should be put together to fabricate actual mechanical components by amorphous films.

In the last decade, some alloys were found to be in the amorphous structure even in

${ }^{*}$ Received 24 Nov., 2010 (No. 10-0554) [DOI: 10.1299/jmmp.5.138]

Copyright $@ 2011$ by JSME 
lower cooling rate and some producing methods were developed for amorphous metals in the form of bulk specimens. In such a circumstance, various products, such as sports equipments, medical devices and so on, have been fabricated by using bulk amorphous alloys [6]. In the case of actual use of amorphous metals for mechanical components, their mechanical properties should be evaluated as fundamental design data. Thus, the mechanical properties of amorphous metals have been investigated by many researchers in recent years [7-9].

However, the most important property for the actual machine design is fatigue strength of the structural materials, since every product should endure during a definite period without failure under cyclic loadings. From this point of view, fatigue tests were performed for single-layer specimens and multi-layer specimens of a Fe-based amorphous alloy in this study. The fundamental $S-N$ property and statistic fatigue property of the amorphous metal were investigated together with their size effect. In addition, the fracture surfaces were observed by scanning electron microscope (SEM) and the fracture mechanisms of this amorphous alloy were discussed from the viewpoint of the fractography.

\section{Material and test method}

The material used in this study was the amorphous metal of $\mathrm{Fe}_{78} \mathrm{~B}_{13} \mathrm{Si}_{9}$ (in atomic percent) which was a long ribbon with the thickness of $25 \mu \mathrm{m}$ and the width of $50 \mathrm{~mm}$. This material was supplied in the reel-wound form by AlliedSignal Company of America. The chemical composition and mechanical properties of this material are shown in Tables 1 and 2, respectively. As indicated in Table 2, Vikers hardness and the tensile strength give high values of $860 \mathrm{HV}$ and $2187 \mathrm{MPa}$, however, the Young's Modulus $57 \mathrm{GPa}$ is extremely smaller than normal crystalline ferrous metals. The specimens of single-layer, ten-layer, and fifty-layer types were prepared to perform the fatigue tests. A lot of single-layer notched specimens were first cut from the long ribbon of the amorphous alloy. Then, many ten-layer specimens were prepared by bonding the single-layer specimens. Fifty-layer specimens were finally obtained by bonding five specimens in the ten-layer type. The adhesive is an epoxy resin and its mechanical properties are shown in Table 3 [10]. Figure 1 shows shape and dimensions of the single-layer specimen whose thickness is $25 \mu \mathrm{m}$. Figure 2 shows the longitudinal section view of ten-layer specimen which is composed of ten single-layer specimens and nine adhesive layers. As mentioned above, five specimens of this type were bonded to prepare a fifty-layer specimen. Every multi-layer specimen was bonded on a horizontal table putting a weight of steel block $(1 \mathrm{~kg})$ during an entire night. The thickness of each adhesive layer in multi-layer specimens is not constant so that sum of thickness of each adhesive layer is in a range of 5\%-28\% for the entire thickness of the multi-layer specimen. Every type of the above specimens has the same round notches at both sides, and the stress concentration factor of each specimen is commonly determined as 1.08 from the handbook by R. E. Peterson [11].

Table 1 Chemical composition of $\mathrm{Fe}_{78} \mathrm{~B}_{13} \mathrm{Si}_{9}$ amorphous metal (mass\%).

\begin{tabular}{c|c|c|c|c|c|c|c|c}
\hline \hline $\mathrm{B}$ & $\mathrm{Si}$ & $\mathrm{C}$ & $\mathrm{Mn}$ & $\mathrm{Al}$ & $\mathrm{P}$ & $\mathrm{S}$ & $\mathrm{Cu}$ & $\mathrm{Fe}$ \\
\hline 2.06 & 5.32 & $0.1>$ & $0.1>$ & $0.1>$ & $0.1>$ & $0.1>$ & $0.1>$ & Bal. \\
\hline
\end{tabular}

Table 2 Mechanical properties of $\mathrm{Fe}_{78} \mathrm{~B}_{13} \mathrm{Si}_{9}$ amorphous metal.

$\left.\begin{array}{c|c|c|c|c}\hline \hline \begin{array}{c}\text { Young's } \\ \text { Modulus }\end{array} & \begin{array}{c}\text { Vickers } \\ \text { Hardness }\end{array} & \begin{array}{c}\text { Thermal } \\ \text { Expansion } \\ \times 10^{-6} \\ \left(1 /{ }^{\circ} \mathrm{C}\right)\end{array} & \begin{array}{c}\text { Tensile } \\ \text { Strength }\end{array} & \begin{array}{c}\text { Crystallization } \\ \text { Temperature }\end{array} \\ \hline \mathrm{E}(\mathrm{GPa}) & \mathrm{HV} & 7.6 & 2187 & (\mathrm{MPa})\end{array}\right)$


Table 3 Mechanical Properties of epoxy resin adhesive (hardened).

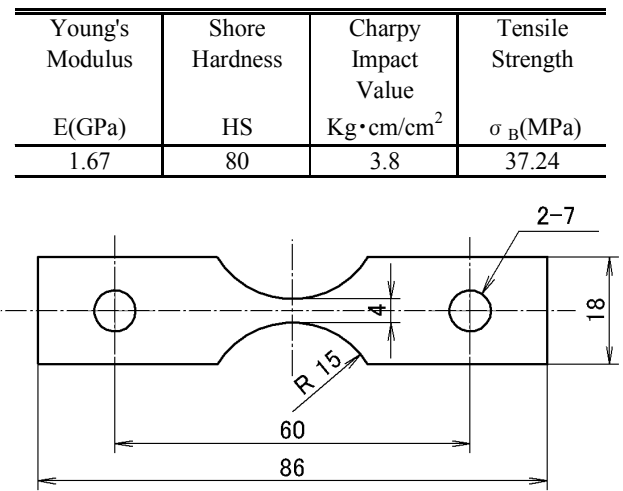

Fig.1 Shape and dimensions of notched specimen.

Adhesive layers

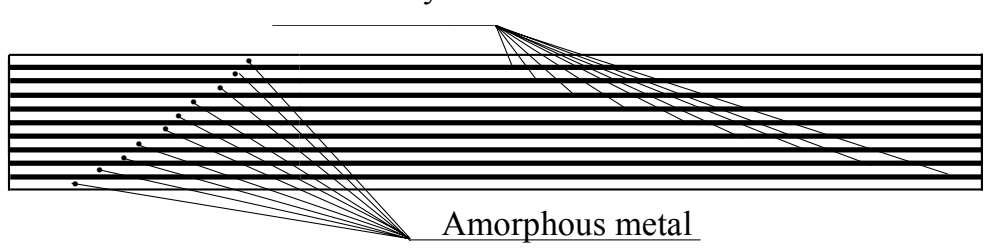

Fig.2 Longitudinal section view of ten-layer notched specimen.

An electro-hydraulic servo testing machine having the loading capacity of $5 \mathrm{KN}$ was used to perform the fatigue tests. Fatigue tests were carried out in tension-tension axial loading at a frequency of $50 \mathrm{~Hz}$ under the stress ratio of $R=0.1$. Here, the applied stresses on ten-layer specimens and fifty-layer specimens were calculated supposing that the load can be carried only by amorphous ribbons without adhesive layers due to the low tensile strength of the adhesive layer comparing with the amorphous metal. The testing environment was in room air without any control of temperature nor humidity.

\section{Experimental results and discussion}

\section{1 $S$ - $N$ property}

Fatigue test data obtained in this study are shown as $S-N$ diagrams in Fig. 3(a)-(c). Fatigue tests were repeatedly performed at each stress level in order to investigate the distribution characteristics of the fatigue life as discussed later. Thus, a number of data points are plotted at the respective stress levels as shown in Fig. 3. In these figures, fatigue lives have large scatter at every stress level and this tendency is more distinctive at lower stress levels. In addition, some specimens can run out at $N=10^{7}$ cycles. Data points attached small arrows indicate the run-out fatigue data. Numbers attached right-hand side of arrows indicate the number of run-out specimens at the corresponding cycles. Solid line in each diagram gives the $S-N$ curve determined by the JSMS standard titled as "Standard Regression Method of $S-N$ curves" [12].

Fatigue strengths at $N=10^{7}$ for the respective types of specimen are given as follows; $\sigma=135 \mathrm{MPa}$ for single-layer specimen, $\sigma=187 \mathrm{MPa}$ for ten-layer specimen, and $\sigma=173 \mathrm{MPa}$ for fifty-layer specimen. In the evaluation of the fatigue strength for metallic materials, fatigue ratio of $\sigma_{\mathrm{w}} / \sigma_{\mathrm{B}}$ (fatigue endurance limit divided by the tensile strength) has been often accepted during a long history of the research on metallic materials. In the case of the present amorphous alloy, the $S-N$ curve tends to decrease continuously with an increase of the fatigue life so that we have no clear knee point in the $S-N$ curve. Accordingly, the 
fatigue strength at $N=10^{7}$ was here accepted to calculate the fatigue ratio instead of the fatigue endurance limit. Values of the fatigue ratio thus obtained for the respective types of specimen are provided by $\sigma / \sigma_{\mathrm{B}}=6.2 \%$ for single-layer specimen, $\sigma / \sigma_{\mathrm{B}}=8.6 \%$ for ten-layer specimen, and $\sigma / \sigma_{\mathrm{B}}=7.9 \%$ for fifty-layer specimen, respectively.

Above values are in a range of $6-9 \%$ and these values are extremely smaller than those for the usual structural steels, in which the fatigue ratio is usually in the range of $40-60 \%$ [13]. Some examples of the fatigue ratio for amorphous alloys were reported by other researchers as 4\% [14], 10\% [15], 16.4\% [16] and 26.5\% [17], respectively. Based on the above comparison, it is found that the fatigue ratios for amorphous alloys are extremely less than those for the usual steels with the crystalline structure. This fact suggests that such amorphous alloys keep superiority in the machine structural use under high static loading, but particular attentions should be paid in the case of cyclic loadings.

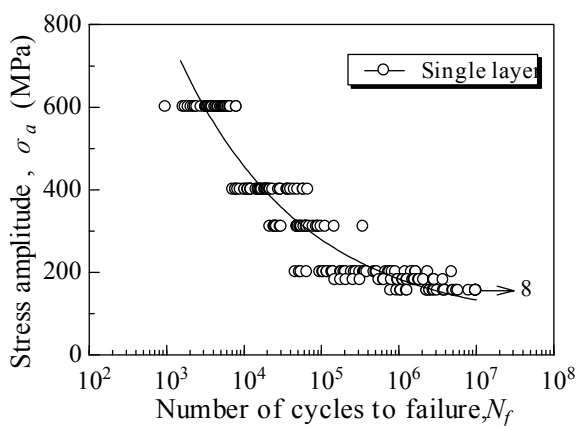

(a) $S$ - $N$ diagram of single-layer specimens

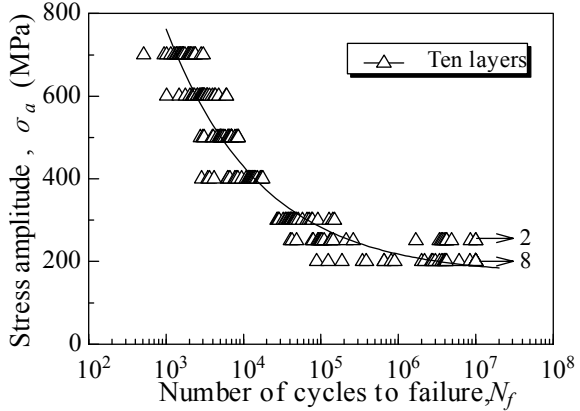

(b) $S$ - $N$ diagram of ten-layer specimens

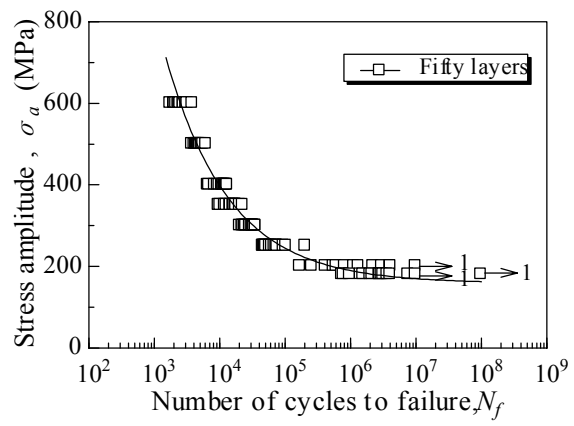

(c) $S$ - $N$ diagram of fifty-layer specimens

Fig.3 $S$ - $N$ diagrams of single-layer, ten-layer and fifty-layer specimens

\subsection{Distribution characteristics of fatigue life}

Weibull distribution has been widely used to evaluate the fatigue life distribution of the structural metallic materials [18-20]. The common Weibull distribution function is expressed as follows [21];

$F(N)=1-\exp \left\{-\left(\frac{N-c}{b}\right)^{a}\right\}$

where, $a, b$ and $c$ are shape parameter, scale parameter and location parameter, respectively. These parameters can be easily obtained by applying the correlation coefficient method [19].

The respective parameters estimated by the correlation coefficient method are shown in Table 4, in which Table (a), (b) and (c) indicate the results for single-layer specimen, ten-layer specimen and fifty-layer specimen, respectively. As explained later, in the case of ten-layer specimens, the fatigue life distribution has two components. Thus, the respective parameters are shown for each component. Fatigue life distributions of singer-layer 
Table 4 Weibull distribution parameters for respective specimens.

(a) Single-layer specimens.

(c) Fifty-layer specimens.

\begin{tabular}{|c|c|c|c|c|c|c|c|}
\hline $\begin{array}{c}\text { Stress } \\
\text { amplitude } \\
\mathrm{MPa}\end{array}$ & $\begin{array}{c}\text { Shape } \\
\text { parameter } \\
a\end{array}$ & $\begin{array}{c}\text { Scale } \\
\text { parameter } \\
b\end{array}$ & $\begin{array}{c}\text { Location } \\
\text { parameter } \\
c\end{array}$ & $\begin{array}{c}\text { Stress } \\
\text { amplitude } \\
\mathrm{MPa} \\
\end{array}$ & $\begin{array}{c}\text { Shape } \\
\text { parameter } \\
a\end{array}$ & $\begin{array}{c}\text { Scale } \\
\text { parameter } \\
b\end{array}$ & $\begin{array}{c}\text { Location } \\
\text { parameter } \\
c \\
\end{array}$ \\
\hline 600 & 2.37 & 4601 & 285 & 600 & 2.05 & 1203 & 1554 \\
\hline 400 & 1.07 & 17080 & 6951 & 500 & 2.74 & 1699 & 3331 \\
\hline 310 & 0.94 & 46944 & 21367 & 400 & 5.79 & 11636 & 6.56 \\
\hline 200 & 0.79 & 433955 & 45457 & 350 & 3.87 & 13373 & 3742 \\
\hline 181 & 1.26 & 1491916 & 62331 & 300 & 2.27 & 13014 & 17562 \\
\hline 155 & 0.85 & 7005340 & 756431 & 250 & 0.69 & 23548 & 45745 \\
\hline & & & & 200 & 0.71 & 1984550 & 152002 \\
\hline & & & & 180 & 0.58 & 3940000 & 743080 \\
\hline
\end{tabular}

(b) Ten-layer specimens.

\begin{tabular}{c|c|c|c|c|c|c|c|c}
\hline & \multicolumn{4}{|c|}{ First life component } & \multicolumn{3}{c}{ Second life component } \\
\hline $\begin{array}{c}\text { Stress } \\
\text { amplitude } \\
\mathrm{MPa}\end{array}$ & $\begin{array}{c}\text { Shape } \\
\text { parameter } \\
a\end{array}$ & $\begin{array}{c}\text { Scale } \\
\text { parameter } \\
b\end{array}$ & $\begin{array}{c}\text { Location } \\
\text { parameter } \\
c\end{array}$ & $p_{1}$ & $\begin{array}{c}\text { Shape } \\
\text { parameter } \\
a\end{array}$ & $\begin{array}{c}\text { Scale } \\
\text { parameter } \\
b\end{array}$ & $\begin{array}{c}\text { Location } \\
\text { parameter } \\
c\end{array}$ & $p_{2}$ \\
\hline 700 & 3.64 & 1909 & 0.51 & 1 & - & - & - & 0 \\
\hline 600 & 2.96 & 3125 & 324 & 1 & - & - & - & 0 \\
\hline 500 & 3.94 & 6201 & 272 & 1 & - & - & - & 0 \\
\hline 400 & 2.55 & 12298 & 2.87 & 1 & - & - & - & 0 \\
\hline 300 & 0.95 & 28200 & 26773 & 1 & - & - & - & 0 \\
\hline 250 & 1.74 & 95400 & 25601 & 0.6 & 1.52 & 5270000 & 1257078 & 0.4 \\
\hline 200 & 0.68 & 6420000 & 75237 & 1 & - & - & - & 0 \\
\hline
\end{tabular}

specimen, ten-layer specimen and fifty-layer specimen are plotted in Fig.4-Fig.6, respectively. From these figures, it is found that the distribution characteristics of the fatigue life are basically well represented by the common Weibull distribution. However, the fatigue life distribution of ten-layer specimens at the stress level of $\sigma_{a}=250 \mathrm{MPa}$ cannot be represented by the common Weibull distribution. In Fig. 5, the dashed line indicates the distribution function obtained by assuming a common Weibull distribution at the stress level of $250 \mathrm{MPa}$. Such a distribution function cannot provide the actual distribution characteristics of the experimental results. Fatigue data at this stress level tend to split into two groups which are the short life group and the long life group. Based on this separation, a horizontal step appears at the cumulative probability level of $F(N) \approx 60 \%$. In such a case, we cannot analyse the distribution property by using the common Weibull distribution.

In order to solve this problem, the following concept of the mixed-mode Weibull distribution having two components is introduced in this study and the distribution function is expressed as follows;

$$
\begin{aligned}
& F(N)=p_{1} F_{1}(N)+p_{2} F_{2}(N) \\
& F_{1}(N)=1-\exp \left\{-\left(\frac{N-c_{1}}{b_{1}}\right)^{a_{1}}\right\} \\
& F_{2}(N)=1-\exp \left\{-\left(\frac{N-c_{2}}{b_{2}}\right)^{a_{2}}\right\}
\end{aligned}
$$


$F_{1}(N)$ is the distribution function for the specimens in the short life group, whereas $F_{2}(N)$ is the distribution function for the specimens in the long life group. $p_{1}$ and $p_{2}$ indicate the occurrence probabilities of the respective distribution components. Of course, $F(N)$ gives the final distribution function in the mixed-mode Weibull distribution and we have $p_{1}+p_{2}=1$.

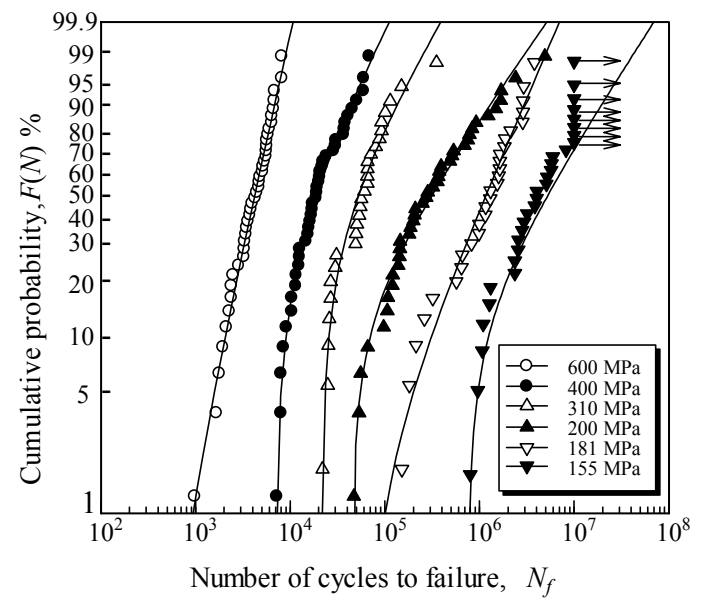

Fig. 4 Fatigue life distribution for single-layer specimens.

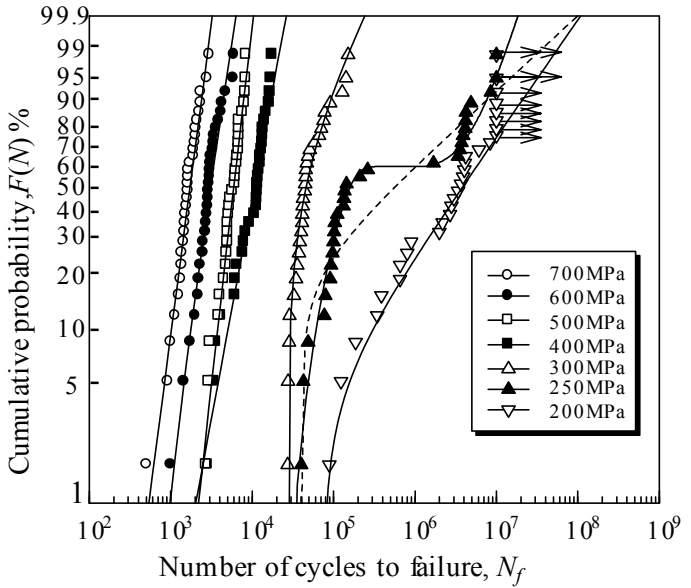

Fig.5 Fatigue life distribution for ten-layer specimens.

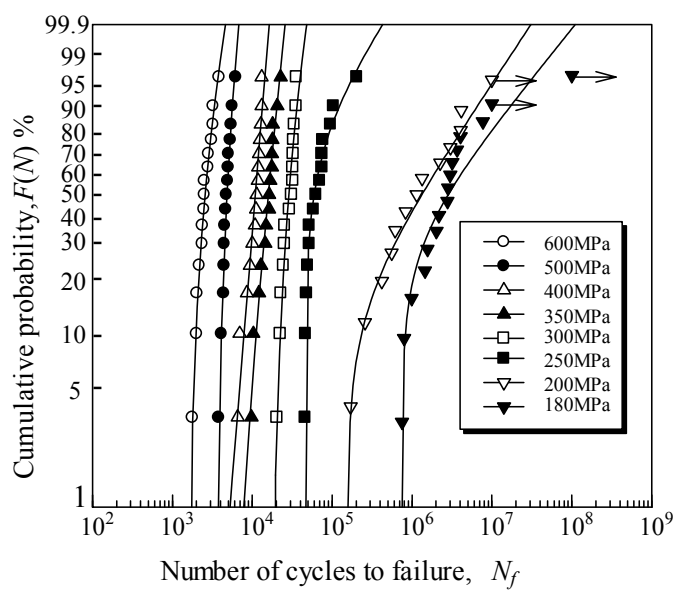

Fig.6 Fatigue life distribution for fifty-layer specimens.

The number of specimens belonging to the short life group is $n_{1}=18$ and the number for the long life group is $n_{2}=12$, respectively. Thus, occurrence probability $p_{1}$ and $p_{2}$ can be 
calculated as follows;

$p_{1}=n_{1} /\left(n_{1}+n_{2}\right)=18 / 30=0.6$ and $p_{2}=n_{2} /\left(n_{1}+n_{2}\right)=12 / 30=0.4$

Substituting distribution parameters and occurrence probabilities in Table 4(b) into Eqs. (2)-(4), we can calculate the distribution function in mixed-mode Weibull distribution. The distribution function thus obtained at $\sigma_{a}=250 \mathrm{MPa}$ is plotted by the solid curve passing through the corresponding data points in Fig. 5, whereas the other dashed curve indicates the distribution curve obtained by assuming the common Weibull distribution. It is clearly found that the distribution function analysed as the mixed-mode Weibull distribution can well represent the distribution characteristics of the actual fatigue life, although a distinct difference is observed for the result calculated by the common Weibull distribution. This fact means that the present analysis based on the mixed-mode Weibull distribution is useful to represent the complicated distribution characteristics of the fatigue life at a definite stress level. But, the reason why the complicated distribution pattern appears at the particular stress level is still unsolved at the present stage. In authors' opinion, this stress level is supposed to be the critical stress under which adhesive layers have an effect on the fatigue life of multi-layer specimen as explained in the last paragraph of Section 3.3.

\subsection{P-S-N property and size effect}

Based on distribution functions of the curves in Fig.4-Fig.6, percentile points at given probabilities of failure can be read at the respective stress levels of the fatigue tests. Thus, percentile points corresponding to failure probabilities of $1 \%, 10 \%, 50 \%, 90 \%$ and $99 \%$ were read at each stress level of the fatigue tests. By connecting these percentile points in each figure, one can obtain the $P-S-N$ curves for each type of the specimen as shown in Fig. $7(\mathrm{a})$, (b) and (c), respectively. In this manner, one can analyse the $P-S-N$ property for any level of the failure probability and the results can be referred as fundamental design data in the actual application of the present materials to the mechanical components.

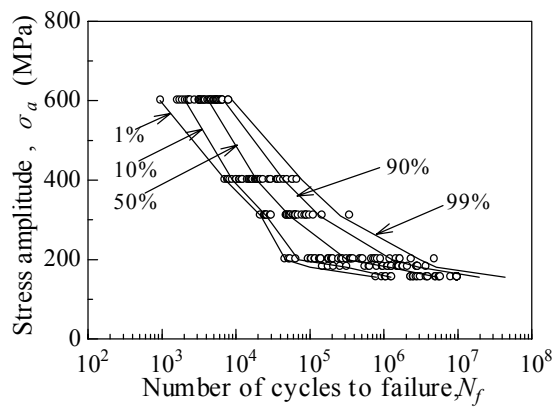

(a) Single-layer specimens

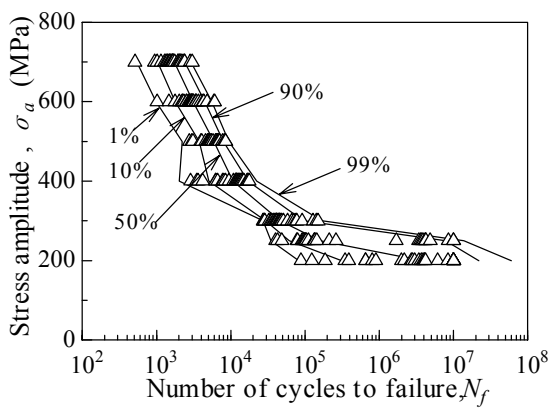

(b) Ten-layer specimens

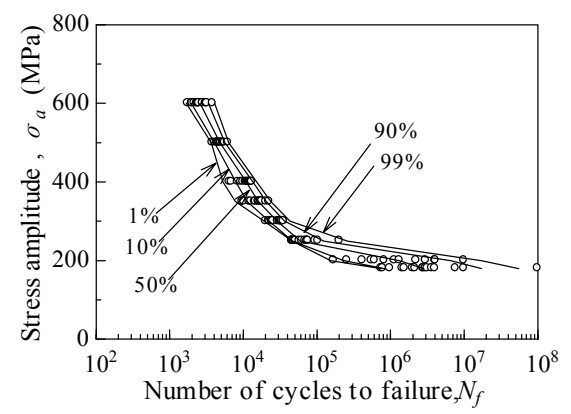

(c) Fifty-layer specimens

Fig. $7 P-S$ - $N$ curves for single-layer, ten-layer and fifty-layer specimens 
In the next place, it is found that the width of the $P-S-N$ curves tends to decrease with an increase of the number of layers. This fact means that the scatter of the fatigue life tends to decrease with an increase of the number of layers. In order to examine such an aspect more precisely, $S$ - $N$ curves for each type of specimen corresponding the respective failure probabilities are plotted in Fig. 8(a)-(e). In cases of low failure probabilities of $1 \%$ and $10 \%, S$ - $N$ curves of each type of the specimens appear at almost same position as seen in Fig. 8(a) and (b). However, the $S$ - $N$ curves for high failure probabilities indicate the size effect so that the specimen consisting of many layers gives a little short life in the life region of $N<10^{5}$. This trend becomes more distinctive at the higher failure probability of $99 \%$ as shown in Fig. 8(e).

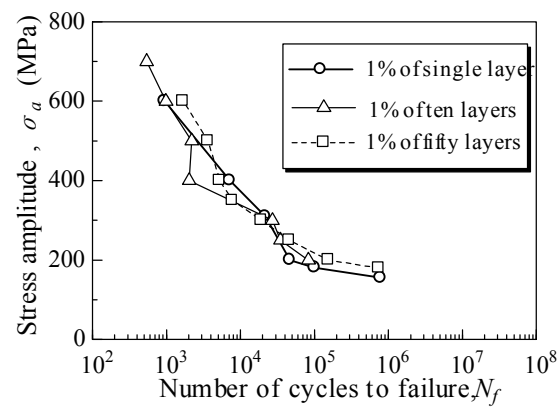

(a) $S$ - $N$ diagram at $1 \%$

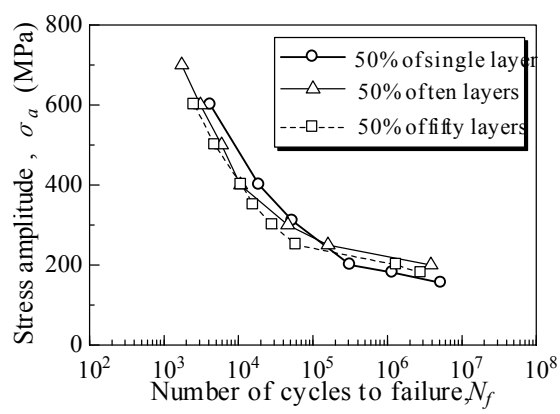

(c) $S$ - $N$ diagram at $50 \%$

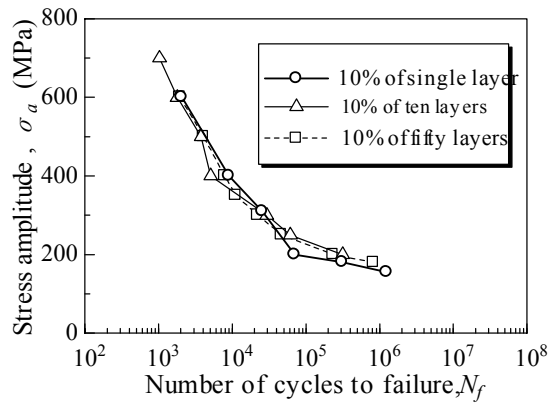

(b) $S$-N diagram at $10 \%$

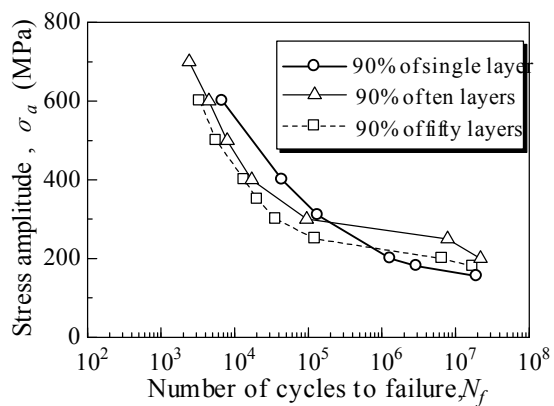

(d) $S$ - $N$ diagram at $90 \%$

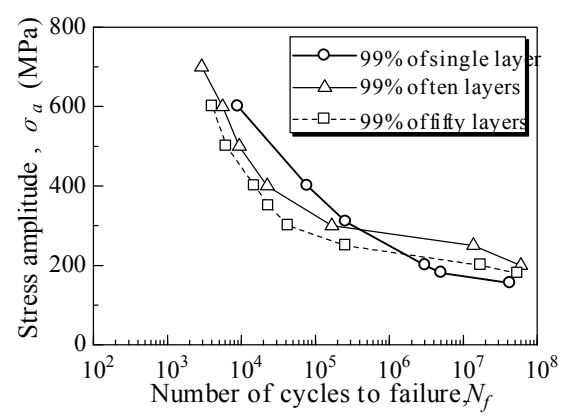

(e) $S$ - $N$ diagram at $99 \%$

Fig. $8 S$ - $N$ diagrams at $1 \%, 10 \%, 50 \%, 90 \%$, and $99 \%$.

Here, let us consider the reason why the above trend appears. Assuming that the fatigue life of the multi-layer specimen is given by the fatigue life of the weakest layer, one can analyse the distribution function of fatigue life for the multi-layer specimen by applying the concept of the weakest link model as follows [22];

$F_{n}(N)=1-\left[1-F_{1}(N)\right]^{n}$ 
where, $F_{1}(N)$ is the distribution function for the single-layer specimen, $n$ is the number of layers and $F_{\mathrm{n}}(N)$ is the distribution function for the multi-layer specimen with $n$ layers, respectively. By differentiating $F_{\mathrm{n}}(N)$ with respect to $N$, we have the probability density function $f_{\mathrm{n}}(N)$ as follows;

$$
f_{n}(N)=\frac{d F_{n}(N)}{d N}=n\left[1-F_{1}(N)\right]^{n-1} f_{1}(N)
$$

where, $f_{1}(N)$ is the probability density function for the single layer specimen. Probability density functions for single-layer specimen and multi-layer specimens (ten-layer specimen and fifty-layer specimen) governed by Eq. (7) are schematically indicated in Fig. 9. Based on this concept, it is found that the lowest bound of the distribution is constant regardless of the number of layers. Another finding is that the mean value of the distribution tends to decrease distinctly with an increase of the number of layers. In addition, percentile points giving high failure probabilities (90\% and 99\%) for multi-layer specimen appear in very short life side rather than the single-layer specimen. This fact is well corresponding to the above experimental trend on the distribution change as shown in Fig. 8(c)-(e).

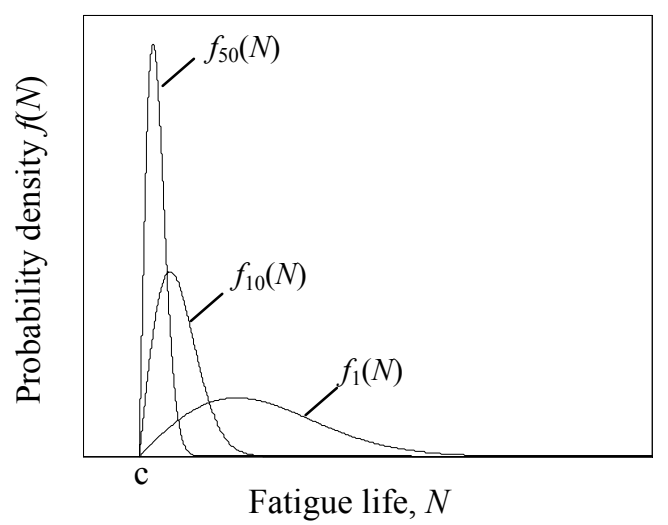

Fig. 9 Probability density functions of variable $N$ for single-layer specimen and multi-layer specimens

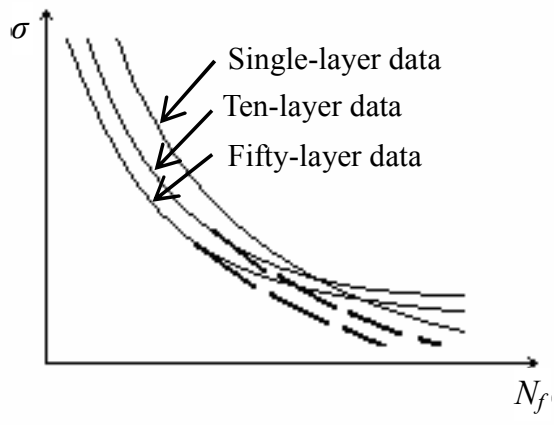

Fig. 10 Illustration of authors' assumption at low stress level

On the other hand, it is found that the fatigue life of multi-layer specimen is longer than the life of single-layer specimen at low stress levels less than $300 \mathrm{MPa}$ as shown in Fig. 8(c)-(e), which means the fatigue life distribution cannot follow the weakest link model at such low stress levels. This fact suggests that some other factors are related to the fatigue life distribution at low stress levels, otherwise $S$ - $N$ diagrams in Fig. 8(c)-(e) should indicate the trend as illustrated in Fig. 10 (dashed lines). The authors have interpreted this aspect as follows: Since the strength level of adhesive layer is not so high, such layers might fail at the early stage of the cyclic loading at high stress level. Thus, the adhesive layers have no effects on the fatigue life of multi-layer specimen at high stress levels and the weakest link 
model can be applied successfully. However, the adhesive layers do not fail at low stress levels and they can carry the load to some extent so that the stress in the amorphous layers becomes a little lower. Accordingly, the fatigue life of the multi-layer specimen becomes longer as illustrated in Fig. 10 (solid lines). This assumption can effectively explain the characteristic life distribution at low stress levels.

\subsection{Fracture surface observation}

Fracture surfaces of failed specimens were observed by SEM. Figure 11 shows typical examples of such observation results for the single-layer specimen failed at the stress amplitude of $200 \mathrm{MPa}$ with the fatigue life of $1.2 \times 10^{6}$ cycles. Figure 11(a) indicates the fracture surface near the crack initiation site, where the crack occurs at the specimen surface and a distinct flat semi-ellipse area is formed near the crack initiation site as to penetrate the thickness of the film specimen. Figure 11(b) shows the fracture surface in the stable fatigue crack propagation region. Arrow in each photograph indicates the crack growing direction. The fracture surface in this region shows a rough morphology covered by similar marks of ' $\mathrm{V}$ ' character, which is called as chevron pattern [23]. Figure 11(c) shows the fracture surface in the final catastrophic fracture region. In this area, the fracture surface exhibits a characteristic pattern like the veins in a leaf of tree, which is called as vein pattern [23].

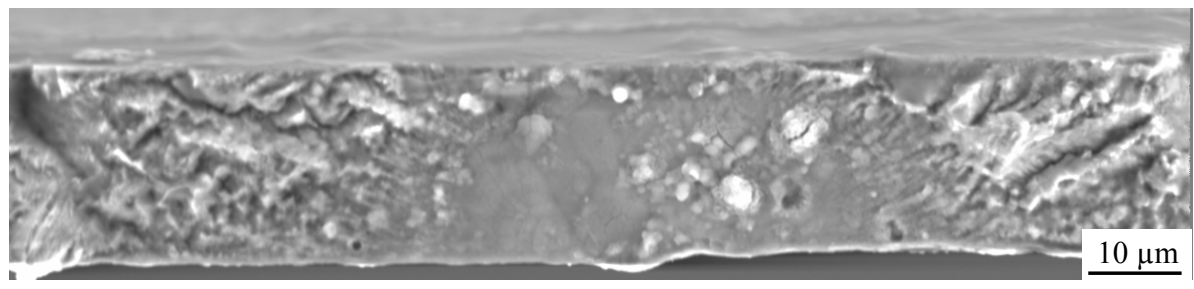

(a) Crack initiation site.

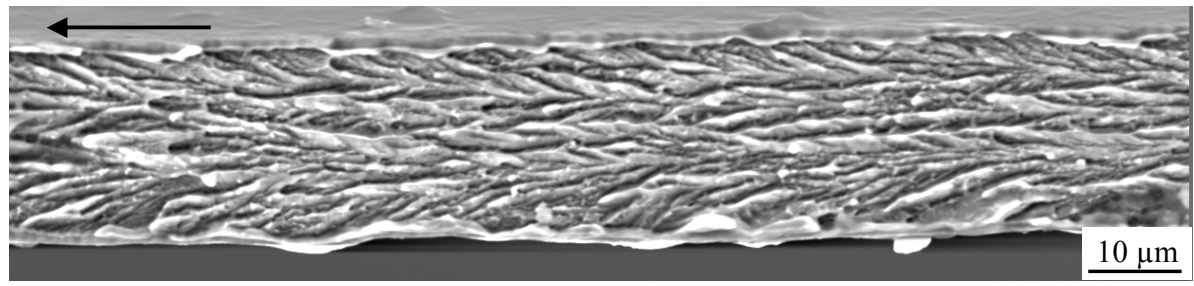

(b) Chevron pattern.

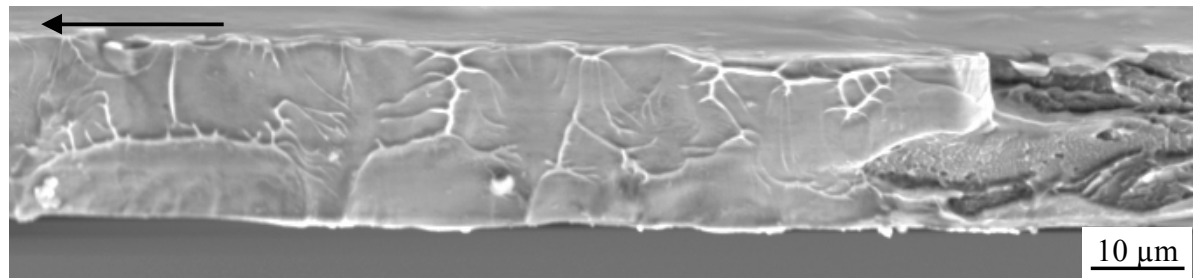

(c) Vein pattern.

Fig.11 Photographs of fracture surface (single layer specimen).

Figure 12 indicates some examples of fracture surfaces for a fifty-layer specimen failed at the stress level of $300 \mathrm{MPa}$ with the fatigue life $2.4 \times 10^{4}$ cycles. In such a multi-layer specimen, a few layers have failed in the mechanism similar to the single-layer specimen. However, the stress of the remaining layers tends to increase due to the reduction of the number of unfailed specimen in the sequence of the cyclic loading. Based on such a circumstance, the remaining layers should fail instantaneously due to the high static load when several layers failed in the mechanism of the fatigue fracture. Fig. 12(a) indicates the 
fracture surface near the crack initiation site in the layer generated the first crack. A flat semi-ellipse area is observed similar to the fracture surface in Fig. 11(a). Fracture surface at the middle stage of the same layer is shown in Fig. 12(b), where the typical chevron pattern was clearly observed corresponding to the pattern in Fig. 11(b). Figure 12(c) indicates an example of the fracture surface of a remaining layer failed in the catastrophic fracture of the multi-layer specimen. This layer is supposed to be failed instantaneously as the specimen fails finally. The entire fracture surface of such a layer is almost covered by the vein pattern peculiar to the static fracture. Of course, this vein pattern was commonly observed on the fracture surfaces of many layers failed at the final fracture of the multi-layer specimen.
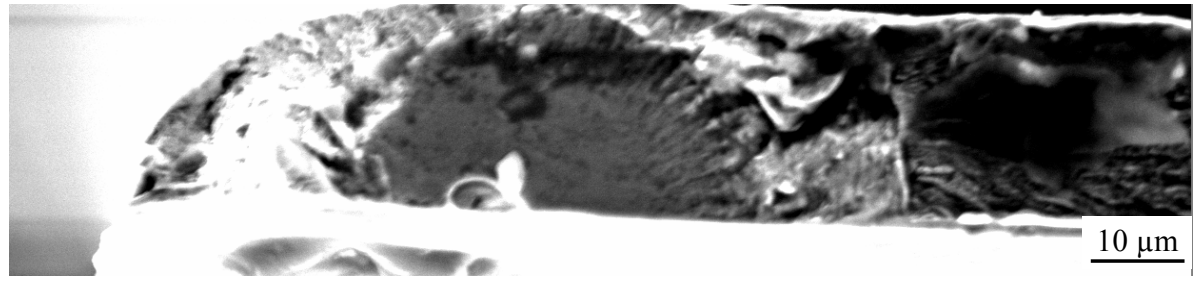

(a) Crack initiation site

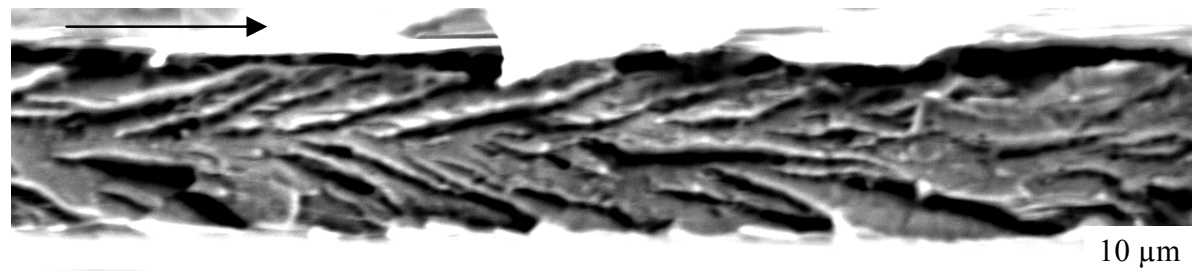

(b) Chevron pattern.

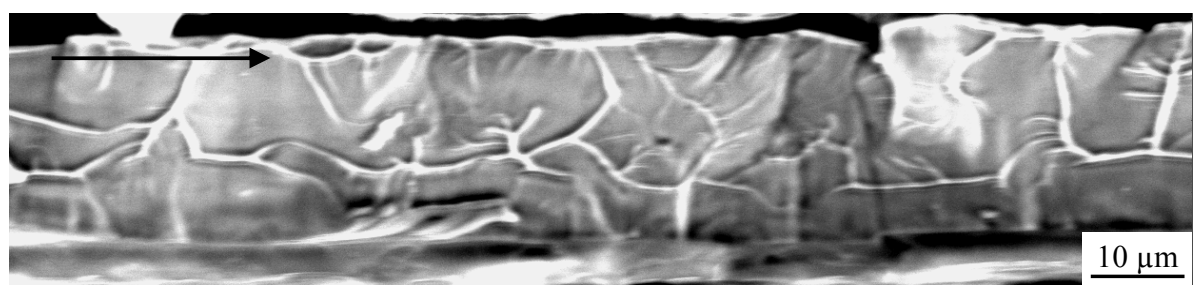

(c) Vein pattern.

Fig.12 Photographs of fracture surface (fifty-layer specimen).

\section{Conclusions}

On the basis of fatigue tests performed on single-layer and multi-layer specimens of $\mathrm{Fe}_{78} \mathrm{~B}_{13} \mathrm{Si}_{9}$ amorphous metal, one can draw the following conclusions on the fatigue properties of the present amorphous metal;

1. The tensile strength of the amorphous metal gives a high value of $2187 \mathrm{MPa}$. However, the fatigue ratio of the amorphous metal is very low as 6-9\%. Thus, a particular attention should be paid in the case of application to the machine structural use under cyclic loadings.

2. Distribution characteristics of the fatigue life for single layer and multi-layer specimens are basically well represented by common Weibull distribution. However, the fatigue life distribution characteristics of ten-layer specimen should be represented by the mixed-mode Weibull distribution at a definite stress level.

3. In the life region of $N<10^{5}$, the multi-layer specimen gives the life a little shorter than the single layer specimen corresponding to the number of layers. This trend can be explained by the weakest link model. However, the trend is found to be not reasonable in the long life region at low stress levels due to the effect of the adhesive layers which can carry the load to some extent. 
4. Fatigue crack occurs at the specimen surface and a flat semi-ellipse area is found near the crack initiation site. In the stable crack initiation region as the penetrated crack, the clear chevron pattern is formed up to the final failure. On the fracture surface in the catastrophic fracture, the vein pattern peculiar to the static fracture of amorphous metals can be widely observed.

\section{References}

(1) Klement, W., Willens, R. H. and Duwez, P., Non-crystalline structure in solidified gold-silicon alloys, Nature, Vol.187 (1960), pp.869-870.

(2) Sakai, T. and Takeuchi, K., Statistical fatigue properties of $\mathrm{Fe}_{78} \mathrm{~B}_{13} \mathrm{Si}_{9}$ amorphous ribbon in cyclic tensile loading, Proc. 5th Japan International SAMPE Symposium, (1997), pp.323-328.

(3) Sakai, T. and Ito, T., A study on tensile strength distribution and its temperature dependence for amorphous alloy ribbons, Journal of the Society of Materials Science, Japan, Vol.47 (1998), pp.1210-1215.

(4) Flores, K. M. and Dauskardt, R. H., Enhanced toughness due to stable crack tip damage zones in bulk metallic glass, Scripta Materialia, Vol.41 (1999), pp.937-943.

(5) Hasegawa, R., Applications of amorphous magnetic alloys in electronic devices, Journal of Non-crystalline Solids, Vol.287 (2001), pp.405-412.

(6) Kakiuchi, H., Inoue, A., Onuki, M., Takano, Y. and Yamaguchi, T., Application of Zr-based bulk glassy alloys to golf clubs, Materials Transactions, Vol.42 (2001), pp.678-681.

(7) Li, X. and Bhushan, B., Measurement of fracture toughness of ultra-thin amorphous carbon film, Thin Solid Film, Vol.315 (1998), pp.214-217.

(8) Maekawa, S., Takashima, K., Shimojo, M., Higo, Y., Sugiura, S., Pfister, B. and Swain, M. V., Fatigue tests of Ni-P amorphous alloy microcantilever beam, Japanese Journal of Applied Physics, Vol.38 (1999), pp.7194-7198.

(9) Takashima, K. and Higo, Y., Fatigue and fracture of a Ni-P amorphous alloy thin film on the micrometer scale, Fatigue and Fracture of Engineering Materials and Structures, Vol.28 (2005), pp.703-710.

(10) Konishi Co., Ltd, Bond Technical Report, (2000), p.4.

(11) Peterson, R. E., Stress concentration design factors, (1953), p.28, John Wiley \& Sons Inc..

(12) Nishikawa, I., Sakai, T., et al., Proposal of standard regression method for S-N curve based on fatigue test data for metallic materials, Journal of the Society of Materials Science, Japan, Vol.51 (2002), pp.54-60.

(13) Schijve, J., Fatigue of Structures and Materials, (2004), p.123, Kluwer Academic Publihers.

(14) Gilbert, C. J., Lippmann, J. M. and Ritchie, R. O., Fatigue of a Zr-Ti-Cu-Ni-Be bulk amorphous metal: stress/life and crack-growth behavior, Scripta Materialia, Vol.38 (1998), pp.537-542.

(15) Katharine, M. F., William L. J. and Reinhold, H.D., Fracture and fatigue behavior of a $\mathrm{Zr}-\mathrm{Ti}-\mathrm{Nb}$ ductile phase reinforced bulk metallic glass matrix composite, Scripta Materialia, Vol.49 (2003), pp.1181-1187.

(16) Qiao, D. C., Liaw, P.K., Fan, C., Lin, Y.H., Wang, G.Y., Choo, H. and Buchanan, R. A., Fatigue and fracture behavior of $\left(\mathrm{Zr}_{58} \mathrm{Ni}_{13.6} \mathrm{Cu}_{18} \mathrm{Al}_{10.4}\right)_{99} \mathrm{Nb}_{1}$ bulk-amorphous alloy, Intermetallics, Vol.14 (2006), pp.1043-1050.

(17) Peter, W. H., Liaw, P. K., Buchanan, R. A. and Liu, C. T., Fatigue behavior of $\mathrm{Zr}_{52.5} \mathrm{Al}_{10} \mathrm{Ti}_{5} \mathrm{Cu}_{17.9} \mathrm{Ni}_{14.6}$ bulk metallic glass, Intermetallics, Vol.10 (2002), pp.1125-1129.

(18) Sakai, T. and Tanaka, T., Estimation of three parameters of Weibull distribution in relation to parameter estimation of fatigue life distribution, Pro. 24th Japan Congress of Materials Research, (1981), pp.141-147.

(19) Sakai, T. and Tanaka, T. Parameter estimation of Weibull-type-fatigue life distributions 
including non-failure probability, Proc. International Conference of FATIGUE'84, Vol. 2 (1984), pp.1125-1137.

(20) Sakai, T., Sakai, T., Okada, K., et al., Statistical fatigue properties of SCM435 steel in ultra-long-life regime based on JSMS database on fatigue strength of metallic materials, International Journal of Fatigue, Vol.28 (2006), pp.1486-1492.

(21) Weibull, W., A statistical distribution function of wide applicability, Journal of Applied Mechanics, Vol.18 (1951), p.293.

(22) Gumbel, E. J., Statistics of Extremes, (1958), p.76, Columbia University Press, New York.

(23) Sakai, T., Fujikawa, T., Takeuchi, K. and Ohuo, M., A study on statistical fatigue properties of multilayer composites made by $\mathrm{Fe}_{78} \mathrm{~B}_{13} \mathrm{Si}_{9}$ amorphous alloy, Journal of the Society of Materials Science, Japan, Vol.52 (2003), pp.2-9. 